\title{
Transformation Digitale de L'Administration Publique au Maroc : Revue de la littérature et état des lieux
}

\author{
Ouajdouni Abdelaziz, Doctorant \\ Chafik Khalid, Enseignant-Chercheur
}

Équipe de Recherche en Management \& systèmes d'information, ENCG de Tanger, Maroc

Boubker Omar, Enseignant-Chercheur

EST de Laâyoune, Université Ibn Zohr, Maroc

Doi:10.19044/esj.2020.v16n19p406 URL:http://dx.doi.org/10.19044/esj.2020.v16n19p406

\section{Résumé}

La transformation digitale aura certes le même impact proéminent en termes d'apport technologique que ce soit dans le secteur public ou privé, mais se met en avant de façon différente dans les deux (02) secteurs. Dans le secteur public, aucune administration publique ne cherche à s'accaparer l'avantage compétitif par rapport à l'autre du fait du monopole détenu par l'état, cependant, les organisations étatiques cherchent à prodiguer un meilleur service aux citoyens/usagers en mettant l'accent sur leur efficacité pérenne advienne que pourra le coût engagé. Ce papier vise à établir une synthèse de la revue de la littérature et de dresser un état des lieux sur la problématique de la transformation digitale de l'administration publique au Maroc.

Mots-clés: Transformation digitale (TD), digitalisation des services publics, gouvernement électronique (e-Gouvernement), Management public 


\title{
Digital Transformation of Public Administration in Morocco: From the Necessity to Seizing the Opportunity
}

\author{
Ouajdouni Abdelaziz, Doctorant \\ Chafik Khalid, Enseignant-Chercheur \\ Équipe de Recherche en Management \& systèmes d'information, \\ ENCG de Tanger, Maroc \\ Boubker Omar, Enseignant-Chercheur \\ EST de Laâyoune, Université Ibn Zohr, Maroc
}

\begin{abstract}
Digital transformation will certainly have the same prominent impact in terms of technological contribution, whether in the public or private sector, but highlighted in different ways in both sectors. As regarding the public sector, no public administration seeks to capture the competitive advantage over others because of the monopoly held by the state. However, state organizations seek to provide a better service to citizens/users by focusing on their long-term efficiency whatever the cost incurred. This paper aims to establish a synthesis of the literature review and to draw up an inventory or status report on the problem of the digital transformation of public administration in Morocco.
\end{abstract}

Keywords: Digital transformation, Digitalization of Public Services, eGovernment (e-Gov), Public Management

\section{Introduction}

Avec l'essor que connaît actuellement l'informatique, l'étendue de l'information et de la communication, généralement connues sous le concept anglais «Information Technology», s'est radicalement modifiée depuis quelques années. Nous vivons actuellement une époque marquée par les innovations dans les technologies de l'information et de la communication (TIC), et par concomitance, des changements possibles dans la gestion des services publics afin de répondre aux attentes des citoyens, qui sollicitent des services numériques en temps réel et de grande valeur (Brown, 2005; Mergel et al. 2019). 
Ceci étant dit, les organisations y compris celles du secteur public se voient obligées de s'adapter aux changements apportés par les nouvelles TIC afin de soutenir leurs objectifs d'affaires (Transparence, interopérabilité et satisfaction des citoyens). C'est dans ce sens que la transformation digitale dans le secteur public intervient. Elle signifie le redéploiement de nouvelles méthodes de travail avec les différentes parties prenantes, de créer de nouveaux cadres de prestations de service et de nouvelles formes relationnelles (Al-Hujran et al., 2015; European Commission \& DirectorateGeneral for Research and Innovation, 2013).

Ces mêmes entreprises du secteur public doivent suivre les évolutions technologiques en optant pour la digitalisation, qui permettrait de faciliter l'implantation d'une « plateforme » organisationnelle structurée (Babinet, 2016).

L'usage des TIC dans le secteur public contribue à l'amélioration du niveau d'efficacité des services et transparence, en améliorant la responsabilisation dans les procédures et la gestion de l'administration publique (Gupta et al., 2008).

Le nouveau management public vise à promouvoir une culture managériale du secteur public axée sur les résultats (Cordella \& Iannacci, 2010). Les TIC sont en effet devenues l'une des solutions les plus couramment mises en œuvre pour standardiser les procédures de travail, d'améliorer le niveau d'efficacité et de transparence des processus organisationnels (Saoud, 2013).

D’une façon globale, nous estimons que les apports des technologies de digitalisation sont importants du fait qu'elles permettent d'être à la fois à jour dans les tâches à exécuter et rapide dans l'utilisation et la diffusion de l'information, entre toutes les parties. En outre, les technologies de digitalisation proposent des avantages assez importants qui pourront modifier les choix organisationnels et managériaux des entreprises du secteur public.

Ces modifications au niveau des choix interviennent dans un contexte qui vise à améliorer d'une manière efficace et efficiente les procédures administratives effectuées par les entreprises du secteur public, afin de produire promptement un produit fini de qualité et d'améliorer le degré de citoyenneté (Bressolles et al., 2014).

$\mathrm{Vu}$ la rareté des publications scientifiques s'attachant à étudier les modalités d'implantation et d'impact des solutions digitales dans le domaine public, notre recherche vise à apporter une lecture terrain de la transformation digitale du secteur public marocain en vue d'accompagner les mégaprojets lancés au Maroc.

L'analyse de ce papier a été réalisée à travers l'analyse de l'état de l'art par le biais d'une synthèse de la revue de la littérature et des données secondaires émanant des administrations publiques marocaines et des rapports 
des organisations à l'échelle internationale. Cela dit, aucun travail empirique n'ayant été réalisé.

Nous essayons de cartographier la digitalisation au niveau des organisations publiques du Royaume en mettant l'accent sur la particularité, les objectifs et les retombées de cet investissement d'envergure. Pour ce faire, le présent papier s'agence en trois (03) axes. Le premier axe est consacré à l'origine du phénomène de la transformation digitale et sa contribution dans le secteur public et privé tout en rapportant les différents profils des pays leaders en matière de la digitalisation. Le deuxième axe se veut comme mis en perspective à l'échelle internationale de la transformation digitale des services publics à travers un benchmark de l'expérience de quelques pays avec la stratégie digitale. Alors que, le troisième axe mettra en avant l'objectif de la TD des secteurs publics ou l'e-Gov ${ }^{13}$ dans le contexte marocain, ses enjeux et l'état des lieux de la TD dans le secteur public ainsi que la cartographie de la digitalisation dans les différentes administrations étatiques.

\section{Transformation digitale : phénomène à plusieurs facettes}

$\mathrm{Ce}$ premier axe s'attache à proposer des définitions de la transformation digitale afin de mieux cerner son concept, d'étudier les différentes facettes du phénomène en questionnant son origine, ses différentes modalités d'implantation dans le secteur public et privé. De même, nous serons en mesure d'apporter une lecture comparative entre les pays pionniers en la matière, en se référant au rapport de la Commission Européenne (2018), et le cas d'un pays arabe en l'occurrence le Royaume Jordanie.

\subsection{Définitions de la digitalisation}

Digitalisation, numérisation, transformation digitale, e-transformation : chaque article utilise l'un ou l'autre de ces concepts pour présenter une évidence, qui se présente dans l'accès des organisations à ce renouveau profond faisant partie intégrante de l'économie digitale qui ponctue le quotidien des individus et qui crée de la valeur ajoutée sur le plan économique.

Gartner $^{14}$ définit la digitalisation comme la mise à profit des technologies numériques pour changer les modèles commerciaux et fournir de nouvelles opportunités de revenus et de création de valeur. Le processus de mise à jour d'une entreprise vers les technologies numériques est évolutif et se produit en effet depuis des décennies. Le processus est rendu possible par une interopérabilité accrue, une transparence de l'information entre départements ministériels et les industries, une assistance et un soutien automatisés et une

\footnotetext{
${ }^{13}$ Par souci de concision et compte tenu de son usage fréquent, nous optons pour le terme « e-Gov » durant cette communication.

${ }^{14}$ Entreprise américaine de conseil et de recherche dans le domaine des techniques avancées.
} 
tendance vers une prise de décision décentralisée. Selon Gartner, l'Internet des objets $(\mathrm{IoT})^{15}$ est considéré comme étant un des piliers majeurs de la digitalisation (Cité par Firouzi et al., 2020).

Elle renvoie à 1'utilisation de nouvelles technologies numériques, telles que les médias sociaux, mobiles (...), en vue d'améliorer l'expérience client, de rationaliser les opérations ou de créer de nouveaux modèles d'affaires (Singh \& Hess, 2017).

Pour Vial (2019, p. 118), la transformation digitale renvoie à «un processus qui vise à améliorer une entité en déclenchant des changements importants dans ses propriétés grâce à des combinaisons de technologies de l'information, de l'informatique, de la communication et de la connectivité ».

Dörner \& Edelman $(2015)^{16}$ ont publié un article qui présente le digital comme un moyen de couverture pour un contexte dans lequel les entreprises et les organisations opèrent. Ils pensent que pour certains cadres, c'est une question de technologie. Pour d'autres, le numérique est une nouvelle façon d'interagir avec les clients. Et pour d'autres encore, cela représente une toute nouvelle façon de faire des affaires. Aucune de ces définitions n'est nécessairement fausse. Cependant, des perspectives aussi différentes s'infiltrent dans les équipes de direction, car elles reflètent un manque de cohérence et de vision consensuelle sur l'orientation stratégique de l'entreprise. Cela se traduit souvent par des initiatives incomplètes ou des efforts malavisés qui conduisent à des opportunités manquées ou des performances médiocres.

La préoccupation principale du présent papier concerne l'egouvernement comme tendance principale de la transformation digitale du secteur public. Cette transformation couvre plusieurs domaines électroniques du secteur public comme la santé, la justice, l'enseignement..., via des plateformes électroniques dédiées à cet effet (e-santé, e-justice, e-learning...).

\subsection{E-Gov: Définition et Origine de l'e-transformation du secteur public}

Avant d'entamer l'origine du phénomène, il est nécessaire de définir le concept de l'e-Gov. Les Nations-Unies (UN) et la société américaine d'administration publique (ASPA) définissent de manière claire ce concept : «Dans son sens large, le gouvernement en ligne inclut l'utilisation de toutes technologies de l'information et de la communication, des télécopieurs aux

\footnotetext{
${ }^{15}$ IBM définit l'IoT comme le concept de connexion de tout appareil (objet physique) à Internet et à d'autres appareils connectés. Il fait référence à la gamme croissante d'appareils connectés à Internet qui capturent ou génèrent une énorme quantité d'informations quotidiennement.

${ }^{16}$ Voir le portail de McKinsey \& Company https://www.mckinsey.com [consulté le 02 Avril, 2020].
} 
ordinateurs de poche Palm sans fil, pour faciliter l'administration quotidienne $d u$ gouvernement. Toutefois, comme le commerce électronique, la vision populaire du gouvernement en ligne n'y inclut que les activités liées à l'Internet Il faudrait ajouter qu'il s'agit d'améliorer l'accès du citoyen à l'information gouvernementale, à ses services et à son expertise de façon à favoriser la participation du citoyen au processus de gouvernement et sa satisfaction envers celui-ci. C'est un engagement permanent du gouvernement envers l'amélioration de la relation entre le citoyen privé et le secteur public au moyen de services, de renseignements et de connaissances améliorés, rentables et efficaces. C'est la réalisation, en pratique, de ce que le gouvernement a de mieux à offrir » (Cité par St-Amant, 2004, p.37).

L'e-Gov est un concept qui a vu le jour en majeure partie, par similitude aux termes et techniques du e-commerce dans le secteur public et représente un service public mis en ligne en faveur du public soit via Internet ou autre dispositif technologique essentiel pour prodiguer des services (Brown, 2005). Cependant, il s'agit d'un concept qui n'est pas limité à l'utilisation d'Internet ou des systèmes accessibles au public pour une utilisation directe par les citoyens/clients/usagers, mais il a commencé comme un domaine afférant aux professionnels du secteur des TIC, réunissant essentiellement des praticiens qui ont pour objectif de relever de nouveaux défis du support Internet en mettant en œuvre de nouveaux systèmes de façon créative (Grönlund \& Horan, 2005).

Le concept e-Gov a vu ces premiers jours à la fin des années 1990, mais l'histoire de l'informatique dans les institutions gouvernementales remonte aux premières apparitions des ordinateurs (Grönlund \& Horan, 2005). Le phénomène a pris de l'avant vers le milieu des années 1980. L'e-Gov est né des interactions entre trois (03) ensembles d'éléments apparents, dont chacun a évolué séparément: les TIC, les concepts de gestion et l'administration proprement dite (Brown, 2005). Selon lui, un facteur notable est que l'innovation technologique et les nouveaux dogmes dans la plupart des pratiques de gestion ont émergé à l'extérieur du secteur publicparticulièrement dans le secteur privé -. Les gouvernements sont largement affectés par des facteurs exogènes, notamment les besoins et les capacités du public. C'est dans ce sens que l'e-gouvernement est toujours un concept de développement global. Alors que les gouvernements s'adaptent de plus en plus à leurs fonctionnalités et à leurs outils, le gouvernement électronique peut continuer à évoluer remarquablement sur plusieurs figurations en l'occurrence, l'évolution technologique, d'administration et de gestion. 


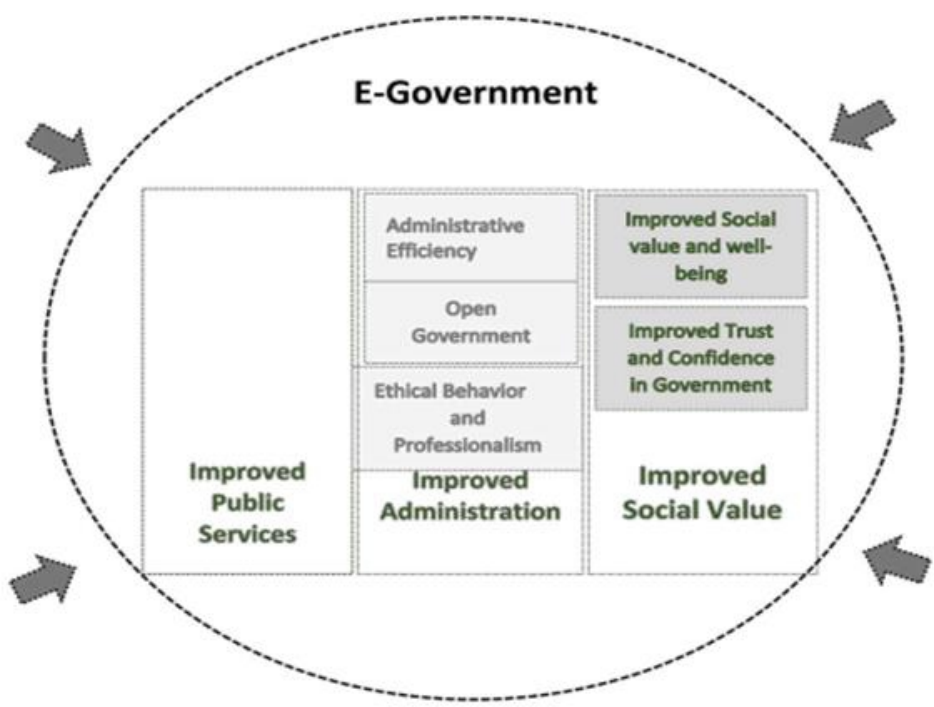

Figure 1. Dimensions de la valeur publique du gouvernement électronique (Twizeyimana \& Andersson, 2019, p. 170)

Une littérature sur les technologies d'information dans les institutions de l'état remonte au moins aux années 1970 (Kraemer \& King, 1986; Rahm, 1999). Cette littérature se penche essentiellement sur l'utilisation des technologies de linformation au sein des institutions gouvernementales, tandis que la littérature récente sur le gouvernement électronique concerne le plus souvent l'utilisation externe ou plus explicitement l'aspect behavioural de l'administration relatif à la satisfaction tel qu'il a été démontré par Herbert Simon (1958) - i.e., les services aux citoyens (Ho, 2002; Kitsios et al., 2019; Oktal et al., 2016).

\subsection{Transformation digitale : Public vs Privé}

Dans une étude menée dans un contexte européen et plus particulièrement hongrois, et compte tenu la finalité de la transformation digitale dans les entreprises analysées tous secteurs confondus, Feher \& Szabo (2018) ont observé un grand écart dans différentes industries. Pour eux, la tendance à la digitalisation est moins évidente d'une industrie à l'autre. Ils rajoutent que les entreprises publiques sont généralement réfractaires à la transformation digitale et ont fait part de leurs attentes concernant l'impact transformationnel de l'informatique à un niveau inférieur à la moyenne, loin derrière les entreprises du marché hongrois. Les organisations publiques n'étant pas fortement affectées par la concurrence, l'urgence de la transformation numérique est moins acceptée. 


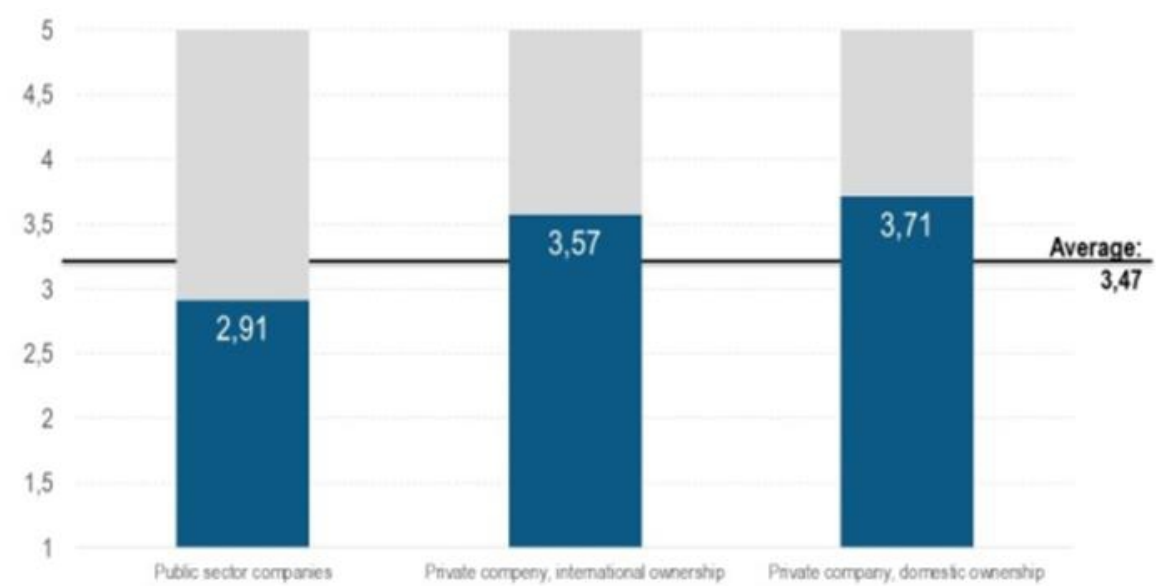

Figure 2. Impact de la transformation digitale dans les organisations hongroises enquêtées

Eggers et Bellman (2015) dans une enquête internationale visant à étudier l'impact de la transformation digitale dans les organisations, soulignent que l'impact des IT sur les services publics est jugé transformateur. Les résultats montrent que les organisations accordent une priorité élevée à la transformation digitale, bien que la voie vers une digitalisation aboutie ne soit pas visible.

\section{Transformation digitale des services publics : mise en perspective à l'échelon international}

La transformation digitale au niveau du service public constitue un point crucial en vue de développer les entreprises publiques et d'améliorer leurs pratiques de gestion dans la majorité des pays de la zone Euro (Calay et al., 2019).

L'ONU soulignait dans son rapport sur l'e-gouvernement à l'appui du développement durable (2016) qu'une tendance mondiale positive tend de plus en plus vers l'innovation et la digitalisation et de plus en plus de gouvernements adoptent les TIC pour offrir des services de qualité aux usagers et d'engager ces derniers dans les processus de prise de décision. Cette enquête octroie de nouvelles raisons que l'administration en ligne a le potentiel de soutenir la mise en œuvre de l'Agenda 2030 et de ses 17 objectifs de développement durable.

Un peu plus loin, l'ONU dans son enquête annuelle sur l'egouvernement dans le monde (2018) mentionnait que l'administration en ligne ne cesse de se développer et que le secteur public devrait prendre des mesures visant à renforcer la solidité des communautés face aux crises en s'appuyant sur des moyens pour les anticiper et réduire leurs influences. 
L'OCDE étudie aussi les enjeux de la transformation digitale dans le secteur public, en mettant l'accent sur le fait que plusieurs pays ont tendance à adopter la solution de numérisation du fait qu'elle permette la dématérialisation des procédures administratives d'ores et déjà en place. Se référant à cette constatation, l'OCDE suggère que les moyens digitaux soient redéployés comme des agents permettant de transformer les administrations et pour améliorer la productivité, mais aussi afin d'améliorer la relation liant l'organisation administrative aux différentes parties prenantes. C'est dans ce sens que l'OCDE a développé des recommandations de la digitalisation du secteur public destinées à ses pays membres. Elles s'agencent autour de deux (02) enjeux importants :

$>$ Mettre en place une transformation digitale efficiente au sein du secteur public en vue de créer une certaine interconnectivité avec les différentes parties prenantes et de les intégrer à la gestion administrative ;

Moderniser le secteur public afin d'améliorer sa productivité.

Nous exposons ci-après l'expérience de quelques pays avec la stratégie technologique, particulièrement en termes de transformation digitale.

\subsection{Cas du Danemark}

Le Danemark a été le premier pays européen et mondial qui a opté pour la digitalisation des services publics depuis des années (Union, 2018). C'est un pays qui a une longue tradition de gouvernance des TIC et élaboration de politiques pour promouvoir l'Internet dans la société (Scupola \& Zanfei, 2016).

Nombreuses sont les études qui ont examiné le développement de du commerce électronique au Danemark. Andersen \& Bjørn-Andersen (2001) ont analysé la stratégie danoise en matière de commerce électronique et d'adoption du commerce électronique et ont constaté l'engagement politique du gouvernement danois à utiliser le commerce électronique à des fins de bienêtre. Pour eux, le commerce électronique devait être utilisé pour développer davantage le modèle actuel de société de bien-être pour une meilleure qualité de vie pour le peuple danois. Ils considèrent qu'un minimum de stress dans le travail constitue une condition sine qua non au développement individuel en vue d'atteindre de nouvelles réalisations que ce soit en science, en soin de santé, en emploi ou en culture.

En outre, Andersen et al. (2003) soulignent que le gouvernement danois s'efforce de faire du Danemark une force mondiale dans les technologies d'information en axant sa stratégie sur le commerce électronique au sein de la société danoise. Scupola \& Zanfei (2016) dans un article sur la transformation numérique dans les bibliothèques universitaires ont fait valoir 
que les politiques d'information qui sont censées améliorer la collaboration entre les différents acteurs publics et privés sont indispensables à la transition vers une approche gouvernance interorganisationnelle dans le secteur public.

Scupola (2018) a précisé dans étude de cas relative à la digitalisation du secteur public danois, les différents agendas de digitalisation du gouvernement danois. Les plus récents sont dédiés au développement digital et à la cybersécurité et sécurité de l'information.

La Commission Européenne dans son rapport sur le tableau de bord de la transformation digitale (2018) a mentionné que le pays a bien performé dans l'infrastructure numérique. Par rapport à 2016, le Danemark s'est nettement amélioré dans les domaines de la transformation digitale, la culture entrepreneuriale, les investissements et l'accès au financement.

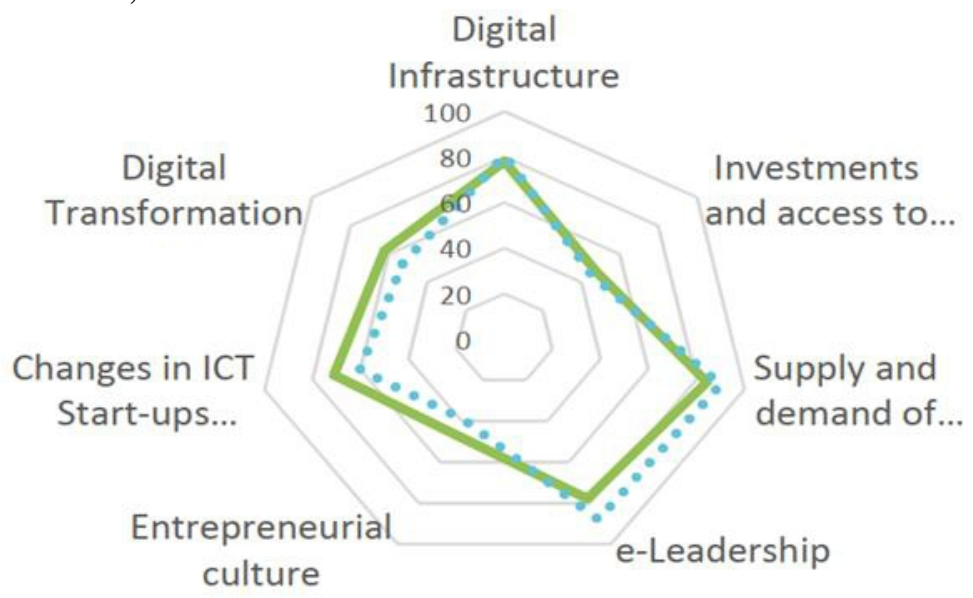

$2017 \quad \cdots \cdots 2016$

Figure 3. Conditions-cadre de la transformation digitale : cas du Danemark

\subsection{Cas de la France}

La France a instauré en 2014 une politique technologique pour l'État qui repose sur trois (03) axes. L'axe premier consiste à harmoniser le système d'information de l'ensemble des administrations du secteur public et garantir une gestion intégrée des données efficace et efficiente. Le deuxième axe s'articule autour de la mise en place d'une plateforme visant à créer une dynamique dans les données administratives destinées aux différentes parties prenantes. Le troisième axe concerne l'e-administration qui sert à dématérialiser les services rendus aux différentes parties prenantes.

La Commission Européenne dans son rapport sur le tableau de bord de la transformation digitale (2018) a établi des conditions-cadres de la transformation digitale afin de classer les pays en se basant sur ces indices. 


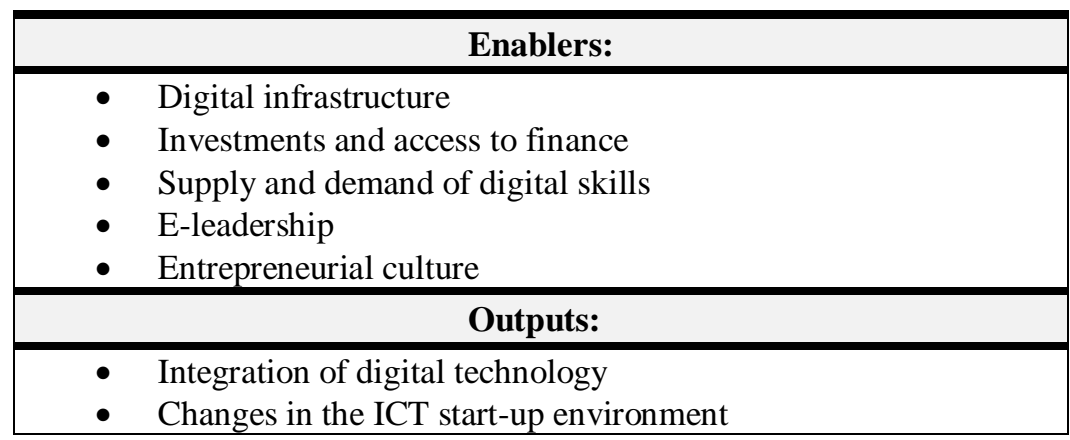

Tableau 1. Conditions-cadres de la transformation digitale

Les principaux atouts de la France résident dans sa culture entrepreneuriale, l'importance de ses investissements et l'accès au financement (Commission Européenne, 2018). Entre 2016 et 2017, la France s'est nettement améliorée en termes de culture entrepreneuriale. Globalement, le profil de la France semble relativement homogène dans la plupart des dimensions à l'exception de son environnement des TIC des start-ups et la transformation digitale, où il est en retard par rapport à d'autres pays de la zone Euro.

Des efforts ont été faits pour intégrer les technologies numériques dans leurs processus de production et adapter les modèles économiques en conséquence (médias sociaux, etc.). En outre, la France dispose d'un cadre financier qui incite l'investissement des entreprises privées dans les TIC. Les entreprises françaises ont un accès facile au financement sur les marchés boursiers et investissent à des niveaux élevés en $\mathrm{R} \& \mathrm{D}$ dans les technologies numériques.

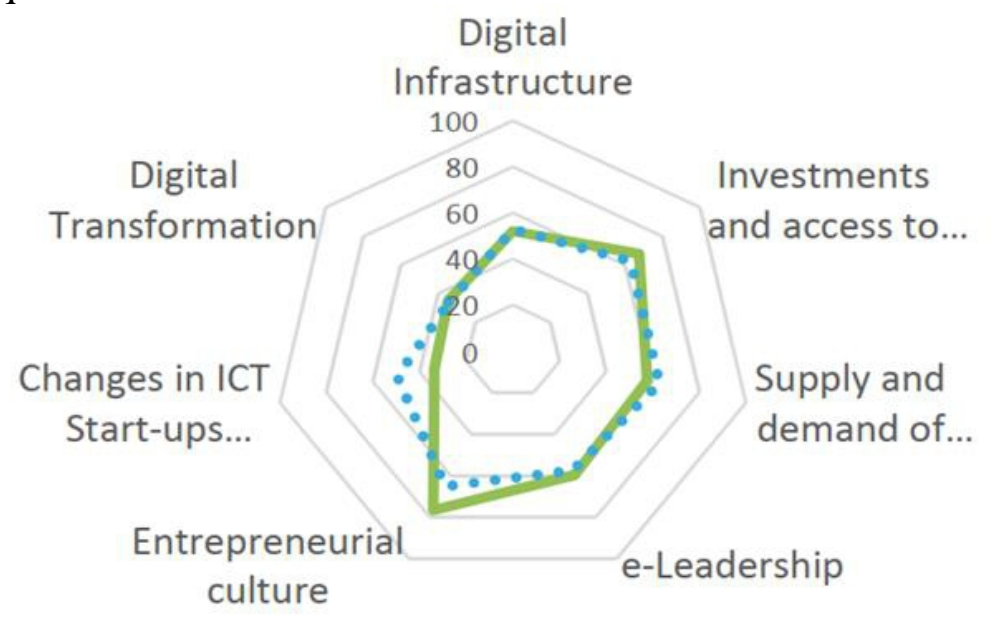

$2017 \quad \cdots 2016$

Figure 4. Conditions-cadres de la transformation digitale : cas de la France 


\subsection{Cas de la Jordanie}

La Jordanie est considérée comme l'un des pays de la région MENA à s'être engagé dans les démarches de bonne gouvernance y compris l'aspect lié aux initiatives entreprises en matière des TIC (Al-Hujran et al., 2015; Ciborra \& Navarra, 2005).

Les efforts redéployés par la Jordanie afin de prodiguer des services en ligne (e-Gov) ont été bel et bien constatés (Al-Hujran et al., 2015; M. K. Alomari et al., 2010). Nonobstant que la démarche d'administration en ligne n'est encore qu'à ses débuts, la Jordanie a pu développer un service d'administration électronique qui est relativement en avant et qui repose l'interaction bidirectionnelle et la démocratie électronique (Al-Hujran et al., 2015; Chatfield \& Alhujran, 2009). En outre, les Nations Unies dans ses rapports de préparation à l'e-Gov (2003 et 2005) ont classé la Jordanie parmi les 5 meilleurs pays arabes en termes d'administration en ligne (Al-Hujran et al., 2015).

Pourtant, l'administration en ligne en Jordanie est confrontée au problème de la faible utilisation des services électroniques mis à disposition (Al-Hujran et al., 2015; Al-Jaghoub et al., 2010; Hujran et al., 2013; Mofleh et al., 2008).

La Jordanie recourt essentiellement aux experts internationaux afin de mettre en exergue les initiatives relatives à l'e-administration (Elsheikh et al., 2008), en les considérant comme de purs projets IT.

En revanche, il ne faut pas omettre le risque de ne pas tenir compte du contexte national de la Jordanie sur le plan culturel, politique, social, traditionnel, éducatif... (Al-Hujran et al., 2015). Au demeurant, Al-Jaghoub et al. (2010) ont précisé que $85 \%$ des citoyens jordaniens n'ont jamais eu accès aux sites Web et à l'e-administration.

Al-Hujran et al. (2015) croient qu'il est indispensable pour le gouvernement jordanien de prendre en considération les aspects sociaux, démographiques, politiques et culturels dans le contexte de l'e-administration afin de réussir ce chantier. Pour eux, la prise en considération de ces facteurs permettrait aux pays y compris la Jordanie de réduire l'écart entre la conception et la réalité de l'e-administration dans le contexte de chaque pays.

Pour le cas de la Jordanie, une ultime compréhension des facteurs qui impactent l'adoption de l'e-administration par les citoyens est d'une importance cruciale. C'est dans ce sens que les améliorations dans ce domaine seraient utiles pour les décideurs publics en ce qui concerne la conception des services en ligne. Concomitamment, il serait utile aux services publics d'améliorer les processus de prestation de services en ligne en vue d'augmenter leurs niveaux d'adoption par les citoyens (Al-Hujran et al., 2015). 


\section{Transformation digitale des services publics : cas du Maroc}

La transformation digitale est aujourd'hui une des tendances clés au sein des organisations publiques et privées du Royaume. Ce troisième axe vise à mettre l'accent sur les objectifs et les enjeux de la transformation digitale au Maroc, en passant par les différentes initiatives et stratégies numériques lancées par le Royaume.

\subsection{Pour quel objectif ?}

L'e-Gov englobe l'ensemble des rôles et des activités de l'administration et repose sur les technologies de l'information et de la communication (TIC). Cet ensemble d'activités de l'administration va bien au-delà d'une comparaison avec le commerce électronique, du fait qu'il regroupe les quatre (04) domaines de la gouvernance et de l'administration publique : les programmes socio-économiques de l'État, ses interactions avec le citoyen et l'État de droit (la démocratie électronique), ses opérations internes et ses relations avec l'environnement international (Brown, 2005).

Selon Brown (2005), il existe quatre (04) aspects de l'e-Gov qui ont des conséquences durables sur le service public : un service axé sur le citoyen, l'information en tant que ressource publique, de nouvelles compétences et relations de travail et des modèles de responsabilité et de gestion.

Les enjeux de l'e-gouvernement sont encore plus importants dans les pays en développement, même s'il offre également des solutions. Dans tous les pays, l'administration publique passe par une nouvelle façon de penser et une nouvelle direction afin de s'assurer que l'e-gouvernement réalise son plein potentiel.

L'administration en ligne a un impact significatif sur l'administration publique en modifiant l'environnement opérationnel des services publics, en introduisant de nouveaux concepts et méthodes dans son fonctionnement et en modifiant la relation entre l'importance relative et les éléments de gestion publique établis. Comme tout le gouvernement, le changement est en cours et il est difficile de prévoir son évolution (Al-Hujran et al., 2015; Hujran et al., 2013).

Le succès des initiatives e-Gov est lié à l'adoption des services en ligne et à l'accès aux services digitalisés par les citoyens (Al-Hujran et al., 2015; Carter \& Bélanger, 2005; Ozkan \& Kanat, 2011). L'amélioration des services en ligne dans le secteur public ne peut se faire sans une utilisation fréquente par les citoyens des services d'administration en ligne.

L'adoption par les citoyens des services de gouvernement électronique est un enjeu important pour le succès des initiatives de gouvernement électronique (Al-Hujran et al., 2015; Carter \& Bélanger, 2005; Liu et al., 2014; Ozkan \& Kanat, 2011). En effet, les services de gestion en ligne n'amélioreront 
pas la prestation de services publics sans leur utilisation par le public (Panagiotopoulos et al., 2019).

Cela soulève la question de savoir comment accroître l'acceptation des citoyens de ces services, en particulier dans les pays arabes. Cependant, à ce jour, peu d'études ont exploré les facteurs qui déterminent l'adoption des services du gouvernement électronique par les citoyens des pays en développement, y compris le Maroc (M. Alomari et al., 2012).

Des recherches antérieures ont subdivisé la littérature existante sur l'utilisation du e-Gov en deux (02) parties (Reddick, 2005). D'une part, la première partie examine l'adoption de l'e-administration du côté relatif à l'offre, qui reflète des éléments importants ou liés aux prestataires de services publics. Jusqu'à présent, cette dimension a exploré les facteurs qui influencent l'adoption et la mise en œuvre de services de gestion en ligne par les organisations gouvernementales (Coursey et al., 2007; Ferro \& Sorrentino, 2010; Li \& Feeney, 2014; Norris \& Moon, 2005; Norris \& Reddick, 2013). Ces facteurs comprennent les caractéristiques organisationnelles (la taille, les formalités administratives, la culture et le soutien de la haute direction), l'infrastructure informatique, les ressources financières et le capital humain qualifié.

D'autre part, la deuxième partie examine l'adoption de l'eadministration du point de vue de la demande, en mettant l'accent sur les « clients » (i.e. les citoyens /usagers) des services publics. Certains chercheurs ont utilisé de nombreux cadres théoriques pour tester l'acceptation par les citoyens des services fournis par le gouvernement (Hujran et al., 2013; Lin et al., 2011; Liu et al., 2014; Rana \& Dwivedi, 2015).

Des exemples de ces facteurs comprennent quant à eux, la confiance, le risque, la sécurité, l'utilité et la facilité d'utilisation, l'attitude, la qualité, la satisfaction et l'expérience informatique.

Néanmoins, bien qu'une grande partie de la littérature se concentre sur l'utilisation de l'e-administration du côté relatif à l'offre, on sait de façon relative, peu de choses sur les raisons et les circonstances instiguant le service public à utiliser les services électroniques (Reddick, 2005). Au demeurant, bien que certaines études préliminaires aient été menées au Maroc pour parler de la stratégie numérique proclamée par le gouvernement en 2010 autrement appelé e-Maroc 2010 et son évolution, tout en passant par les méthodes de développement et redéploiement de l'e-Gov ou même la conception d'un cadre en vue d'implémenter un gouvernement électronique personnalisé (E1 Aichi, 2012; Kettani et al., 2008; Rochdi, 2001; Zaoui et al., 2014, 2019), elles ont ignoré l'impact des facteurs culturels et sociopolitiques sur les décisions des Marocains à opter pour l'e-administration.

Lytras \& de Pablos (2011) ont souligné que le développement d'une société de la connaissance nécessite des citoyens informés et actifs en raison 
de leur attitude positive et de leur utilisation pratique des technologies innovantes telles que l'e-administration. Par conséquent, malgré que AlHujran et al. (2015) dans leur article, n'abordent pas directement la contribution des citoyens au développement de la société de la connaissance, ils ont mis l'accent sur l'impact positif sur le développement de la société de la connaissance par les gouvernements et les citoyens grâce à l'adoption et à l'utilisation de l'e-administration et la démocratisation des services en ligne.

En tant que visionnaire avisé, le roi Mohammed VI a introduit les technologies de l'information dans l'administration publique pour devenir un enjeu majeur dans l'amélioration de l'efficacité de l'information, l'amélioration de la qualité des services et le soutien des réformes structurelles. SM le Roi a insisté sur l'e-administration au service de l'usager dans son discours à l'occasion de l'ouverture de la 1re session de la $1^{\text {re }}$ année législative de la $10^{\mathrm{e}}$ législature en octobre 2016, « [...] Par ailleurs, l'administration électronique doit être généralisée selon une approche intégrée permettant aux différents départements et aux divers services un accès commun aux informations. De fait, l'utilisation des nouvelles technologies contribue à faciliter l'accès, dans les plus brefs délais, du citoyen aux prestations, sans qu'il soit nécessaire de se déplacer souvent à l'Administration et de s'y frotter, car c'est là la cause principale de l'extension du phénomène de corruption et du trafic d'influence ». Conscient de l'utilité que revêt le chantier de la transformation digitale dans l'essor de l'administration publique marocaine, le souverain a placé les technologies de l'information au cœur du programme de réforme administrative de manière cohérente et réalisable.

Le conseil économique, social et environnemental (CESE) dans son rapport $^{17}$ publié en 2019 , relatif à sa contribution au chantier de réflexion autour du nouveau modèle de développement du Maroc, a mis l'accent sur la transformation digitale comme une locomotive indéniable afin de réconcilier la confiance du citoyen et faire du Maroc un pays solidaire et prospère pour tous ses citoyens. Le CESE pense que la transformation numérique constituerait la base d'une nouvelle génération de services publics efficaces et accessibles axés sur l'autonomisation des diverses parties prenantes, autrement dit, une des avenues préconisées pour augmenter l'implication et la responsabilité de la population en matière du e-Gov.

L'Agence de Développement du Digital (ADD), l'instance étatique chargée de la mise en œuvre de la stratégie de l'état en matière de développement du digital, a approuvé la note dictant les orientations générales pour le développement du digital au Maroc à l'horizon 2025. L'ADD a défini

${ }^{17}$ Voir le portail du Conseil Économique, Social et Environnemental. « Le nouveau modèle de développement du Maroc : contribution du Conseil Économique, Social et Environnemental » (2019) http://www.cese.ma/ [consulté le 23 mars, 2020]. 
les perspectives de développement du digital au Maroc en axant les interventions sur les principaux objectifs suivants :

- Une administration digitale au service des citoyens et des entreprises ;

- Une économie compétitive grâce aux gains de performance amenés par un écosystème digital et innovation ;

- Une société inclusive grâce au digital ${ }^{18}$.

Le plan national de la réforme de l'administration (2018-2021) vise l'instauration d'une administration performante au service du citoyen-usager. Il s'agit d'une réforme qui recèle en soi tant de projets considérables dispatchés suivant quatre axes de transformation structurelle à savoir la transformation organisationnelle, managériale, digitale et morale. Ce plan vise à rendre l'action publique plus limpide et plus propice, conciliant la société civile avec l'administration via l'adoption de nouvelles façons de concertation.

\subsection{Digitalisation des services publics : Choix ou nécessité ?}

Force est de constater que la Constitution de juillet 2011 instigue l'état à garantir aux citoyens l'exercice plein et égal de leurs droits civils et politiques, ainsi que leurs droits fondamentaux qu'ils soient économiques, sociaux, culturels et environnementaux. L'efficacité de cette catégorie de droits dite de "nouvelle génération" repose sur la disponibilité d'une infrastructure propice et accessible à toutes et à tous sans aucune discrimination.

C'est dans ce sens que la digitalisation des services publics constitue une nécessité de premier plan, invitant les pouvoirs publics à penser à la conjuguer en choix stratégiques tous secteurs étatiques confondus. Dans son rapport, le CESE étant son rôle consultatif, recommande à l'état de prendre les mesures nécessaires afin de concrétiser sa volonté d'instaurer une nouvelle génération de services publics performants et accessibles à tous les usagers. Pour ce faire, cinq axes stratégiques sont indispensables à savoir:

- Veiller à ce que les services de santé de haute qualité soient fournis à l'ensemble de la population, au bénéfice de tous les citoyens;

- Garantir aux citoyens le droit à une protection de la justice intègre, fiable et efficace qui protège leur dignité, leur liberté et leurs droits fondamentaux;

- Veiller à ce que les citoyens aient droit à des services de transports publics étendus, sécures et de qualité;

- Assurer l'accès à un logement et à un cadre de vie décent;

- Prioriser les activités culturelles et sportives en investissant dans le développement des infrastructures, des équipements et des talents.

${ }^{18}$ Voir le portail de l'Agence de Développement du Digital https://www.add.gov.ma/ [consulté le 02 Avril, 2020]. 


\section{3. État des lieux de la transformation digitale des services publics au Maroc}

Dans ce paragraphe, nous exposons les éléments-clés de l'état des lieux (initiatives et stratégies numériques lancées au Maroc) et les orientations possibles de la transformation digitale des services publics au niveau du Royaume.

Les technologies d'information et de communication (TIC) constituent un outil indispensable permettant le développement sur le plan socioéconomique. Comme d'autres pays, le Maroc s'est engagé dans un plan de restructuration du secteur des technologies de l'information en promulguant en 1996 la loi n ${ }^{\circ} 24-96$ relative à la poste et aux télécommunications ${ }^{19}$, qui a été considéré comme un prélude à la première libéralisation de l'industrie des télécommunications. Dans ce contexte, le Maroc a adopté diverses stratégies numériques : le plan quinquennal 1999-2003, e-Maroc 2010 qui s'étale sur la période 2005-2010 et la stratégie Maroc Numéric 2013 (MN2013) couvrant la période 2009-2013.

L'évaluation des services publics en ligne (Plan quinquennal 19992003, e-Maroc 2010 ou MN2013) implique des services fournis par le secteur public sur Internet, quel que soit leur degré de dématérialisation. En outre, l'objectif de telles initiatives est de mettre en œuvre des services publics digitalisés axés sur le citoyen/client/usager visant à resserrer les liens entre ce dernier et l'administration publique, via une intégration amplifiée et une diffusion généralisée des TI au niveau de tous les acteurs sociaux (État, administrations publiques, entreprises et usagers).

À titre de rappel laconique, le plan quinquennal 1999-2003 conçu et formulé par l'ex SEPTTI ${ }^{20}$, a octroyé la priorité au développement des télécommunications et des nouvelles technologies comme priorités nationales du Maroc et choix stratégique pour favoriser le développement économique, industriel et social du Royaume. Les principaux objectifs de ce plan quinquennal s'articulaient autour des points suivants: -Élaborer une législation qui traite de la protection des noms de domaine commerciaux, des données personnelles et des problèmes de commerce électronique; -Amorcer un tournant décisif au niveau de l'éducation par l'intégration des outils informatiques et Internet dans les écoles; -Éviter les fractures numériques grâce à des politiques qui encourageraient le déploiement de nouvelles technologies dans les régions et les segments de population les plus pauvres; -Prodiguer des financements pour favoriser la création des entreprises dans l'ère de l'économie dite de connaissance.

\footnotetext{
${ }^{19}$ Version consolidée de la loi n ${ }^{\circ} 24-96$ relative à la poste et aux télécommunications https://www.anrt.ma/ [consulté le 23 mars, 2020].

${ }^{20}$ Secrétariat d'État auprès du premier ministre chargé de la poste et des Technologies des Télécommunication et de l'Information.
} 
La stratégie e-Maroc 2010 visait à développer l'économie de la connaissance au Maroc et reposait sur un partenariat public / privé « dynamique et coordonné » qui s'articule autour de deux (02) objectifs stratégiques essentiels et étroitement liés, en réduisant la fracture numérique et en positionnant le Maroc à l'échelle internationale compte tenu ses caractéristiques économiques, culturelles et géostratégiques.

La stratégie MN2013 quant à elle, s'articulait autour de quatre (04) axes à savoir :

- L'accès des citoyens à l'Internet haut débit et promouvoir la communication et l'accès à la connaissance ;

- L'amélioration des services publics par le biais de programmes d'administration en ligne pour rapprocher l'administration des besoins des utilisateurs en termes d'efficacité, de qualité et de transparence ;

- L'encouragement des PME à informatiser leurs processus pour augmenter la productivité ;

- Le développement de l'industrie $\mathrm{TI}^{21}$.

Ce qui importe ici, c'est l'appréciation de la mise en œuvre des services en ligne et la contribution de ceux-ci dans l'amélioration des services publics, avec pour toile de fond le rapprochement de l'administration aux citoyens/usagers.

La Cour des comptes a souligné dans son rapport ${ }^{22}$ sur l'évaluation de la stratégie MN2013 que l'analyse des différents plans digitaux qu'a connus le Royaume à partir de 1999 a permis de relever un certain affaiblissement des efforts en matière de redéploiement et de mise en œuvre, exprimé essentiellement par l'instabilité de l'autorité gouvernementale chargée de la gestion du secteur des TIC, ce qui a eu un impact négatif sur la mise en œuvre, la capitalisation des expériences afférentes au domaine en termes d'exécution et de traçabilité des différents documents liés à l'achèvement des projets. Selon le même rapport, il convient de noter que les évaluations des divers plans numériques ont été reportées sine die et qu'aucune mise au point sur les résultats et identification des faiblesses et des facteurs entravant leur mise en œuvre n'ont été dès lors révélées. Il y a lieu de noter qu'avant même d'atteindre le terme fixé par la stratégie e-Maroc 2010 qu'une nouvelle stratégie a été lancée. Par conséquent, sans rime ni raison, il a été observé des discontinuités flagrantes entre les stratégies autour des mêmes finalités.

\footnotetext{
${ }^{21}$ Voir le portail du programme e-gouvernement du Royaume du Maroc, rapport sur la Stratégie Nationale pour la Société de l'Information et de l'Économie Numérique http://www.egov.ma/ [consulté le 23 mars, 2020].

${ }^{22}$ Voir le portail de la cour des comptes, rapport sur l'évaluation de la stratégie Maroc Numéric 2013, Rapport particulier n 05/13/CH IV http://www.courdescomptes.ma/ [consulté le 23 mars, 2020].
} 
La Cour des comptes dans le même rapport d'évaluation du MN2013, souligne le faible avancement des projets e-Gov et la non réalisation de quelques projets structurants tels que l'identifiant unique des citoyens, l'identifiant unique des entreprises ou le projet Gateway qui consiste à mettre en place une plateforme d'interopérabilité intégrée au niveau du programme e-Gov qui faisait partie des projets de la stratégie gouvernementale e-Maroc 2010 qui s'intitule « One Gov». Il a fallu attendre le discours royal du 29 juillet 2018 à l'occasion du 19e anniversaire de l'accession du Souverain au Trône pour que le gouvernement puisse concrétiser réellement ce projet Gateway pour qu'il soit techniquement opérationnel afin de repenser rationnellement le mode de délivrance des services publics administratifs.

Le Souverain a précisé dans son discours que : «[...] et empêchant, d'autre part, toute administration publique de demander, de la part d'un investisseur potentiel, des documents ou des informations qui sont déjà en possession d'une autre administration publique. C'est, en effet, aux services publics qu'il revient d'organiser un échange coordonné des informations, grâce au recours à l'informatique et aux nouvelles technologies ».

La réalisation de cet objectif nécessite la mise en place de deux (02) piliers, le premier consiste à partager les données entre administrations et ce via l'échange d'informations entre deux (02) administrations, l'une octroyant une information importante à une autre pour l'aboutissement d'une démarche demandé par le citoyen, et/ou via la création de bases de données fédérées pour le partage et la collaboration, donnant aux gestionnaires de la chose publique l'accès à des données crédibles, à jour, et complètes sur le citoyen demandeur du service ou de la démarche en question. Le deuxième pilier est de simplifier le processus de gestion en remplaçant les pièces justificatives demandées par les citoyens en accédant à une base de données partagée ou en communiquant avec l'administration qui est censée délivrer la pièce justificative qui fait l'objet de demande via une plateforme sécurisée d'échanges d'informations " Gov to Gov » en vue de vérifier l'authenticité des informations fournies par l'usager et qui sont vérifiables à travers la Gateway ${ }^{23}$. Grosso modo, c'est un principe clé ou le citoyen ne doit communiquer des données d'ordre personnel qu'une seule fois, ce qui implique un véritable partage obligatoire des données entre les différentes administrations du secteur public.

Dans un autre rapport qui s'intitule «évaluation des services publics en ligne $»^{24}$, la Cour des comptes a procédé à une analyse du positionnement du Maroc à l'échelon international en matière de l'e-Gov. Elle a relevé plusieurs constats. Durant la période 2010-2017, des avancées remarquables en matière des services en ligne ont été réalisées dans certaines administrations

${ }^{23}$ Appelé aussi le « Once-Only »

${ }^{24}$ Voir le portail de la cour des comptes, rapport sur l'évaluation des services publics en ligne -synthèse- http://www.courdescomptes.ma/ [consulté le 24 mars, 2020]. 
telles que les impôts, la douane et le commerce extérieur ainsi que la conservation foncière.

L'évaluation de la disponibilité en ligne et de la maturité des principaux services est basée sur les références annuelles de la Commission Européenne en matière d'e-Gov. Quant à l'ouverture des données publiques, son évaluation est basée sur l'utilisation des données brutes et les rapports de l'Open Data Barometer publiés par la World Wide Web Foundation ${ }^{25}$. Selon le même rapport, en termes de développement des services en ligne, bien que le Maroc se soit développé dans le classement des Nations Unies (du 140e en 2008 au $82^{\mathrm{e}}$ en 2014), cette évolution n'a pas perduré.

En 2018, le Maroc a fortement chuté, se classant ainsi dans le $78^{\mathrm{e}}$ dans l'indice des services en ligne et $110^{\mathrm{e}}$ rang dans l'indice de l'e-Gov. En outre, concernant les deux (02) autres composantes de l'e-gouvernement, à savoir le capital humain et l'infrastructure informatique, le niveau du Maroc n'a pas éprouvé un réel changement, il est resté à un niveau faible à l'échelle internationale : le Royaume se positionne dans le $104^{\mathrm{e}}$ rang dans l'infrastructure informatique et $148^{\mathrm{e}}$ rang dans le capital humain. Cela constitue un obstacle à l'utilisation généralisée des services en ligne développés par dans le secteur public.

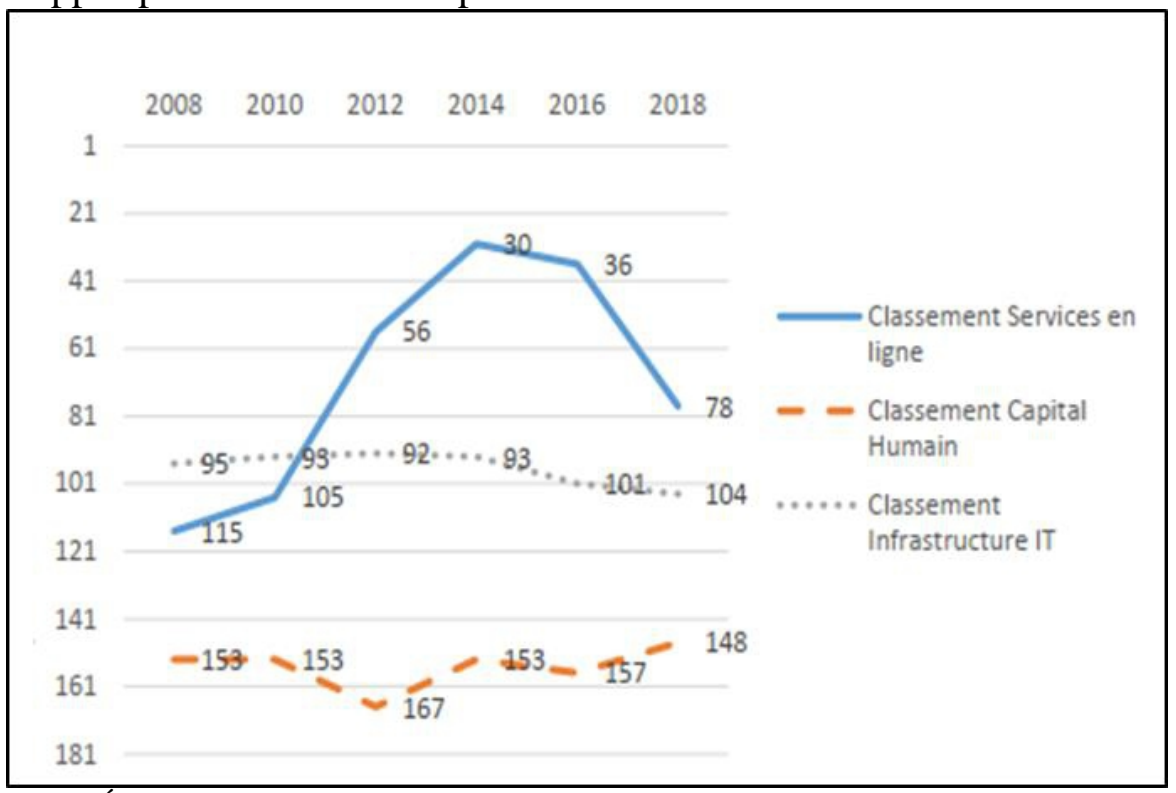

Figure 5. Évolution du classement du Maroc sur les indices « Services en ligne», « Capital humain » et $«$ Infrastructure IT $»^{26}$

${ }^{25}$ Organisation à but non lucratif créée par Tim Berners Lee en 2009 pour promouvoir un Web ouvert. Elle publie l'Open data barometer pour mesurer l'avancée et l'impact de l'open data.

${ }^{26}$ Rapport sur l'évaluation des services publics en ligne synthèse, http://www.courdescomptes.ma/ [consulté le 24 mars, 2020]. 
La Cour des comptes a également souligné que, malgré les progrès réalisés par le Maroc dans le classement des Nations Unies entre 2012 et 2016 sur les services en ligne, la disponibilité en ligne de certains services importants n'a pas suivi les ambitions annoncées dans le plan e-Gov couvrant 2009-2013. C'est le cas, par exemple, des services liés à l'état civil, à la création en ligne des entreprises et à l'immatriculation des voitures en ligne.

Le tableau ci-après présente les différents services en ligne en termes de maturité électronique allant d'un faible niveau à un bon niveau de maturité.

\begin{tabular}{|c|c|}
\hline Bon niveau de maturité & Niveau de maturité faible à moyen \\
\hline $\begin{array}{ll}> & \text { Impôts sur les sociétés } \\
> & \text { TVA } \\
> & \text { Déclaration en douane } \\
> & \text { Impôts sur le revenu } \\
> & \text { Contributions sociales pour les employés } \\
> & \text { Recherche d'emploi } \\
> & \text { Inscription à un cycle de l'enseignement } \\
& \text { supérieur } \\
> & \text { Marchés publics }\end{array}$ & 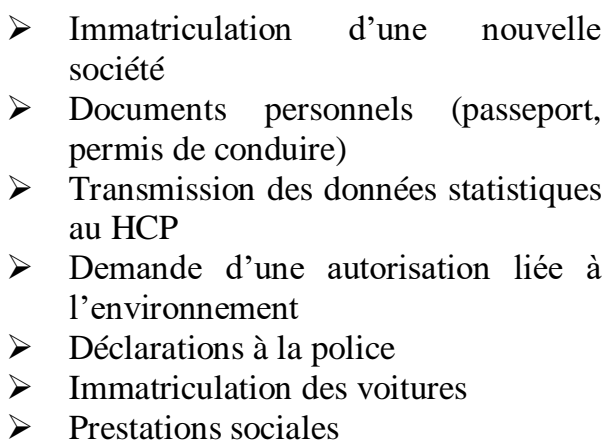 \\
\hline
\end{tabular}

Tableau 2. Maturité des principaux services en ligne ${ }^{27}$

\section{Conclusion et voies futures de la recherche}

L'adoption de la numérisation des procédures tend à se généraliser dans les organisations qu'elles soient publiques ou privées, et que sa mise en place nécessite de ses organisations qu'elles puisent dans leurs efforts afin de faire face aux enjeux financiers, humains et stratégiques tout en sachant que le retour sur investissement n'est pas facile à appréhender. Une organisation et plus particulièrement dans le secteur public, ne peut se transformer d'un jour à l'autre. Cela peut dépendre de la culture organisationnelle et de la réticence des différents acteurs à l'adaptation aux pratiques digitalisées.

Toute chose est égale par ailleurs, par conséquent, il existe un risque inhérent à la transformation digitale dans le secteur public, ce qui nécessite l'adoption de mesures prudentielles et perspicaces.

En vue de profiter au maximum des opportunités prodiguées par les solutions IT, il est indispensable de mettre en place des technologies nouvelles et de faire adapter davantage l'humain à son utilisation. A contrario, de telles initiatives de transformation digitale dans le secteur public demeurent incohérentes et inutiles. Au demeurant, les administrations et entreprises du secteur public devraient opter pour une transformation qui va en parallèle avec

\footnotetext{
${ }^{27}$ Rapport sur l'évaluation des services publics en ligne synthèse, http://www.courdescomptes.ma/ [consulté le 24 mars, 2020].
} 
la culture organisationnelle de chaque pays, cela dit, il faut approprier la procédure de digitalisation au contexte du secteur public pour identifier les axes stratégiques et défis à relever et attribuer des technologies à forte valeur ajoutée pour une cartographie de digitalisation intégrée et réussie. À cet égard, l'appel à la mise en place des pratiques New Public Management est primordial en vue de favoriser la transformation digitale dans les organisations publiques.

$\mathrm{Au}$ Maroc, nous pouvons constater que l'état à lancer plusieurs chantiers de digitalisation des administrations publiques ${ }^{28}$. Si le Royaume a pris du retard dans le domaine de la digitalisation de son secteur public, il a de bons atouts à faire valoir.

Avec la crise sanitaire que connait le monde actuel (Covid 19), la digitalisation du secteur public devient plus que jamais une nécessité du premier ordre, afin d'alléger certaines procédures et d'améliorer l'accès des citoyens/usagers aux services publics (Santiso \& Bernard, 2019), à travers la gestion des données de façon efficace et efficiente et la dématérialisation des procédures administratives, et bâtir une administration digitalisée promotrice d'une nouvelle génération IT.

Il est important de souligner que le bien-être des citoyens constitue d'un des critères d'évaluation de l'action publique (Offredi \& Laffut, 2013), cela reste valable pour la politique de digitalisation des administrations publiques. La question qui se pose à ce niveau est d'identifier les modalités d'influence de la transformation digitale «e-Gov» sur l'amélioration de la qualité de service et l'accroissement du sentiment de citoyenneté des individus. Nous nous attellerons à cette question dans les travaux ultérieurs.

\section{References:}

1. Al-Hujran, O., Al-Debei, M. M., Chatfield, A., \& Migdadi, M. (2015). The imperative of influencing citizen attitude toward e-government adoption and use. Computers in Human Behavior, 53, 189-203. https://doi.org/10.1016/j.chb.2015.06.025

2. Al-Jaghoub, S., Al-Yaseen, H., \& Al-Hourani, M. (2010). Evaluation of awareness and acceptability of using e-government services in developing countries: The case of Jordan. The Electronic Journal Information Systems Evaluation, 13(1), 1-8.

3. Alomari, M. K., Sandhu, K., \& Woods, P. (2010). Measuring Social Factors in E-government Adoption in the Hashemite Kingdom of Jordan. International Journal for Digital Society, 1(2), 123-134. https://doi.org/10.20533/ijds.2040.2570.2010.0017

\footnotetext{
${ }^{28}$ http://www.egov.ma/fr/ [Consulté le 02 Avril, 2020, à 10h00].
} 
4. Alomari, M., Woods, P., \& Sandhu, K. (2012). Predictors for egovernment adoption in Jordan: Deployment of an empirical evaluation based on a citizen-centric approach. Information Technology \& People, 25(2), 207-234. https://doi.org/10.1108/09593841211232712

5. Andersen, K. V., \& Bjørn-Andersen, N. (2001). Globalization and Ecommerce: Growth and Impacts in Denmark.

6. Andersen, K. V., Bjørn-Andersen, N., \& Dedrick, J. (2003). Governance Initiatives Creating a Demand-Driven E-Commerce Approach: The Case of Denmark. The Information Society, 19(1), 95105. https://doi.org/10.1080/01972240309475

7. Bressolles, G., Durrieu, F., \& Senecal, S. (2014). A consumer typology based on e-service quality and e-satisfaction. Journal of Retailing and Consumer $\quad$ Services, 21(6), 889-896. https://doi.org/10.1016/j.jretconser.2014.07.004

8. Brown, D. (2005). Le gouvernement électronique et l'administration publique. Revue Internationale des Sciences Administratives, Vol. 71(2), 251-266.

9. Calay, V., Mosty, M., \& Paque, R. (2019). La digitalisation de l'administration publique wallonne. État des lieux et perspectives (No. 29; pp. 1-108). Institut wallon de l'évaluation, de la prospective et de la statistique.

10. Carter, L., \& Bélanger, F. (2005). The utilization of e-government services: citizen trust, innovation and acceptance factors*. Information Systems Journal, 15(1), 5-25. https://doi.org/10.1111/j.13652575.2005.00183.x

11. Chatfield, A. T., \& Alhujran, O. (2009). A cross-country comparative analysis of e-government service delivery among Arab countries. Information Technology for Development, 15(3), 151-170. https://doi.org/10.1002/itdj.20124

12. Ciborra, C., \& Navarra, D. D. (2005). Good governance, development theory, and aid policy: Risks and challenges of e-government in Jordan. Information Technology for Development, 11(2), 141-159. https://doi.org/10.1002/itdj.20008

13. Commission Européenne (2018). Digital Transformation Scoreboard 2018. EU businesses go digital: Opportunities, outcomes and uptake. Publications Office of the European Union, Luxembourg, DOI, 10(691861), 10-2826.

14. Cordella, A., \& Iannacci, F. (2010). Information systems in the public sector: The e-Government enactment framework. The Journal of Strategic Information Systems, 19(1), 52-66. https://doi.org/10.1016/j.jsis.2010.01.001 
15. Coursey, D., Yang, K., Kasserkert, A., \& Norris, D. (2007). E-Gov Adoption in US Local Governments: Bridging Public Management and Institutional Explanations in a Pooled Time Series Modellcopyright. 2007 Public Management Research Conference, Tucson, Arizona.

16. Eggers, W. D., \& Bellman, J. (2015). The journey to government's digital transformation Deloitte UK. Deloitte United Kingdom.

17. El Aichi, M. M. E. (2012). Moroccan e-government ten years' evolutions. Proceedings of the 6th International Conference on Theory and Practice of Electronic Governance, 492-493. https://doi.org/10.1145/2463728.2463832

18. Elsheikh, Y., Cullen, A., \& Hobbs, D. (2008). e-Government in Jordan: challenges and opportunities. Transforming Government: People, Process and Policy, 2(2), 83-103. https://doi.org/10.1108/17506160810876176

19. European Commission, \& Directorate-General for Research and Innovation. (2013). Powering European public sector innovation: towards a new architecture : report of the expert group on public sector innovation. Publications Office.

20. Feher, P., \& Szabo, Z. (2018). Digitalization in the Public Sector Findings of a Hungarian Survey. 2018 12th International Conference on Software, Knowledge, Information Management \& Applications (SKIMA), 1-6. https://doi.org/10.1109/SKIMA.2018.8631534

21. Ferro, E., \& Sorrentino, M. (2010). Can intermunicipal collaboration help the diffusion of E-Government in peripheral areas? Evidence from Italy. Government Information Quarterly, 27(1), 17-25. https://doi.org/10.1016/j.giq.2009.07.005

22. Firouzi, F., Farahani, B., Weinberger, M., DePace, G., \& Aliee, F. S. (2020). IoT Fundamentals: Definitions, Architectures, Challenges, and Promises. In F. Firouzi, K. Chakrabarty, \& S. Nassif (Eds.), Intelligent Internet of Things (pp. 3-50). Springer International Publishing. https://doi.org/10.1007/978-3-030-30367-9_1

23. Grönlund, А., \& Horan, T. A. (2005). Introducing e-Gov: History, Definitions, and Issues. Communications of the Association for Information Systems, 15. https://doi.org/10.17705/1CAIS.01539

24. Gupta, B., Dasgupta, S., \& Gupta, A. (2008). Adoption of ICT in a government organization in a developing country: An empirical study. The Journal of Strategic Information Systems, 17(2), 140-154. https://doi.org/10.1016/j.jsis.2007.12.004

25. Ho, A. T.-K. (2002). Reinventing Local Governments and the EGovernment Initiative. Public Administration Review, 62(4), 434444. https://doi.org/10.1111/0033-3352.00197 
26. Hujran, O. A., Aloudat, A., \& Altarawneh, I. (2013, April 1). Factors Influencing Citizen Adoption of E-Government in Developing Countries: The Case of Jordan [Article]. International Journal of Technology and Human Interaction (IJTHI). www.igiglobal.com/article/content/77917

27. Kettani, D., Moulin, B., \& Mahdi, A. E. (2008). Proposition of a Method for the Development and Deployment of e-Government Systems that Emphasize Good Governance. 2008 International MCETECH Conference on E-Technologies (Mcetech 2008), 227-231. https://doi.org/10.1109/MCETECH.2008.35

28. Kitsios, F., Tsotoulidou, A., \& Kamariotou, M. (2019). User Satisfaction and Acceptance of the e-justice system in Greece. 7.

29. Kraemer, K. L., \& King, J. L. (1986). Computing and Public Organizations. Public Administration Review, 46, 488-496. JSTOR. https://doi.org/10.2307/975570

30. Li, M.-H., \& Feeney, M. K. (2014). Adoption of Electronic Technologies in Local U.S. Governments: Distinguishing Between EServices and Communication Technologies. The American Review of Public Administration, 44(1), 75-91. https://doi.org/10.1177/0275074012460910

31. Lin, F., Fofanah, S. S., \& Liang, D. (2011). Assessing citizen adoption of e-Government initiatives in Gambia: A validation of the technology acceptance model in information systems success. Government Information Quarterly, 28(2), 271-279. https://doi.org/10.1016/j.giq.2010.09.004

32. Liu, Y., Li, H., Kostakos, V., Goncalves, J., Hosio, S., \& Hu, F. (2014). An empirical investigation of mobile government adoption in rural China: A case study in Zhejiang province. Government Information Quarterly, 31(3), 432-442. https://doi.org/10.1016/j.giq.2014.02.008

33. Lytras, M., \& de Pablos, P. O. (2011). Software Technologies in Knowledge Society J.UCS Special Issue. 3.

34. Mergel, I., Edelmann, N., \& Haug, N. (2019). Defining digital transformation: Results from expert interviews. Government Information $\quad$ Quarterly, 101385. https://doi.org/10.1016/j.giq.2019.06.002

35. Mofleh, S., Wanous, M., \& Strachan, P. (2008). The gap between citizens and e-government projects: the case for Jordan. Electronic Government, an International Journal, 5(3), 275-287.

36. Norris, D. F., \& Moon, M. J. (2005). Advancing E-Government at the Grassroots: Tortoise or Hare? Public Administration Review, 65(1), 64-75. https://doi.org/10.1111/j.1540-6210.2005.00431.X 
37. Norris, D. F., \& Reddick, C. G. (2013). Local E-Government in the United States: Transformation or Incremental Change? Public Administration Review, 73(1), 165-175. https://doi.org/10.1111/j.1540-6210.2012.02647.x

38. Offredi, C., \& Laffut, M. (2013). Le bien être peut il être un critère d'évaluation de l'action publique ? Revue française d'administration publique, 148(4), 1003-1016. https://doi.org/10.3917/rfap.148.1003

39. Oktal, O., Alpu, O., \& Yazici, B. (2016). Measurement of internal user satisfaction and acceptance of the e-justice system in Turkey. Aslib Journal of Information Management, 68(6), 716-735. https://doi.org/10.1108/AJIM-04-2016-0048

40. Ozkan, S., \& Kanat, I. E. (2011). e-Government adoption model based on theory of planned behavior: Empirical validation. Government Information Quarterly, 28(4), 503-513. https://doi.org/10.1016/j.giq.2010.10.007

41. Panagiotopoulos, P., Klievink, B., \& Cordella, A. (2019). Public value creation in digital government. Government Information Quarterly, 36(4), 101421. https://doi.org/10.1016/j.giq.2019.101421

42. Rahm, D. (1999). The role of information technology in building public administration theory. Knowledge, Technology \& Policy, 12(1), 74-83. https://doi.org/10.1007/s12130-999-1015-3

43. Rana, N. P., \& Dwivedi, Y. K. (2015). Citizen's adoption of an egovernment system: Validating extended social cognitive theory (SCT). Government Information Quarterly, 32(2), 172-181. https://doi.org/10.1016/j.giq.2015.02.002

44. Reddick, C. G. (2005). Citizen interaction with e-government: From the streets to servers? Government Information Quarterly, 22(1), 3857. https://doi.org/10.1016/j.giq.2004.10.003

45. Rochdi, N. (2001). e-Maroc: la transition du Maroc vers l'économie de l'information et du savoir. Les Cahiers du numerique, 2(3), 251265.

46. Santiso, C., \& Bernard, M. (2019). Révolution numérique et transformation de l'action publique. Politique étrangère, Été (2), 129. https://doi.org/10.3917/pe.192.0129

47. Saoud, H. (2013). Les Technologies de l'information et de la communication comme levier de transformation du management des organisations publiques [Thesis, Versailles-St Quentin en Yvelines].

48. Singh, A., \& Hess, T. (2017). How Chief Digital Officers promote the digital transformation of their companies. MIS Quarterly Executive, $16(1)$.

49. Scupola, A. (2018). A Case Study of Digital Transformation of Danish Public Services: Actors and Policies. 2018 11th CMI International 
Conference: Prospects and Challenges Towards Developing a Digital Economy within the EU, 14-18. https://doi.org/10.1109/pctdde.2018.8624818

50. Scupola, A., \& Zanfei, A. (2016). Governance and innovation in public sector services: The case of the digital library. Government Information Quarterly, 33(2), 237-249. https://doi.org/10.1016/j.giq.2016.04.005

51. St-Amant, G. (2004). E-Gouvernement: cadre d'évolution de l'administration électronique - ProQuest.

52. Twizeyimana, J. D., \& Andersson, A. (2019). The public value of EGovernment - A literature review. Government Information Quarterly, 36(2), 167-178. https://doi.org/10.1016/j.giq.2019.01.001

53. Union, P. O. of the E. (2018, January 12). eGovernment Benchmark 2017 : taking stock of user-centric design and delivery of digital public services in Europe: final background report. Volume 2. [Website]. Publications Office of the European Union.

54. Vial, G. (2019). Understanding digital transformation: A review and a research agenda. The Journal of Strategic Information Systems, 28(2), 118-144. https://doi.org/10.1016/j.jsis.2019.01.003

55. Zaoui, I., Elmaghraoui, H., Chiadmi, D., \& Benhlima, L. (2014). Towards a Personalized E-Government Platform. 6.

56. Zaoui, I., Lamharhar, H., Chiadmi, D., \& Benhlima, L. (2019). A personalized e-Gov framework to bridge silos between Moroccan administrations. Proceedings of the ArabWIC 6th Annual International Conference Research Track, 1-7.

https://doi.org/10.1145/3333165.3333183 